\title{
Inverse Modeling of Mass-conservative Numerical Model for Variably Saturated Seepage Flow
}

\author{
Tomoki Izumi ${ }^{1}$, Masayuki Fujihara ${ }^{2}$, Junichiro Takeuchi ${ }^{3}$ and Toshihiko Kawachi ${ }^{4}$
}

\begin{abstract}
An inverse method for identifying the unsaturated hydraulic conductivity in variably saturated seepage flow model is proposed. As the basic equation which governs the forward problem, the mixed-form Richards equation is considered which is conservative in mass balance describing the flow in terms of pressure head and moisture content. For functional representation of the relative hydraulic conductivity (RHC) which is a major unknown parameter to be identified, the free-form parameterized function (or sequential piecewise cubic spline function) which provides high flexibility in identifying the functional form of the parameter is employed. To determine values of the coefficients of the function in the manner that errors between the observed and computed pressure heads are minimized, a simulationoptimization algorithm with the aid of the Levenberg-Marqurdt method is constructed. The method proposed is applied to in-situ soil column in an upland crop field, and its validity is examined in terms of reproducibility of desorption process in the soil. The results show that the simulation-optimization algorithm is successful in finding RHC of functional form, and that the forward solution model with the RHC parameter so identified could well reproduce actual desorption process when air-temperature dependency of the observed pressure head variation is appropriately eliminated.
\end{abstract}

Keywords: Inverse analysis; Soil hydraulic property; Free-form parameterization; Mixed-form Richards equation

\section{Introduction}

For sustainable development, it is indispensable to predict and/or assess impacts of human activities on the environment. A numerical analysis is one of the great powerful tools for the prediction and assessment of such problems. Though a numerical analysis is required to reproduce a physical phenomenon of interest more accurately, the accuracy of a numerical analysis depends on the model structure given by a governing equation and the model parameters included in the governing equation. Therefore to select an appropriate governing equation and to determine the adequate model parameters are of great importance to construct a dependable simulation model.

The governing equation mostly used in a subsurface water flow analysis is Richards equation which includes as unknown parameters the soil hydraulic properties (i.e. the soil water retention curve and unsaturated hydraulic conductivity). Richards equation can be expressed in different three forms according to the state variables solved: a pressurehead-based ( $\psi$-based) form, a moisture-content-based $(\theta$ based) form, and a mixed form. Since the $\theta$-based form is not applicable for the saturated zones where the hydraulic diffusivity takes infinite values, the $\psi$-based form which can express all possible ranges of saturation is often used. However Celia et al. (1990) reported that numerical models which

\footnotetext{
${ }^{1}$ Assistant Professor, Faculty of Agriculture, Ehime University, 3-5-7 Tarumi, Matsuyama, Ehime 790-8566, Japan (Corresponding Author) Email: t_izumi@agr.ehime-u.ac.jp

${ }^{2}$ Professor, Faculty of Agriculture, Ehime University, 3-5-7 Tarumi, Matsuyama, Ehime 790-8566, Japan

${ }^{3}$ Assistant Professor, Graduate School of Agricultural science, Kyoto University, Kitashirakawa-oiwake-cho, Sakyo-ku, Kyoto 606-8502, Japan

${ }^{4}$ Professor, Graduate School of Agricultural science, Kyoto University, Kitashirakawa-oiwake-cho, Sakyo-ku, Kyoto 606-8502, Japan
}

solve $\psi$-based form produce significant errors in mass balance, and proposed an alternative model which solves the mixed form perfectly conserving the mass. After the work of Celia et al. (1990), the mixed form of Richards equation is often used as the governing equation for seepage flow, and the computational efficiency of its numerical model is improved (Huang et al., 1996). Almost all the numerical models proposed by Celia et al. (1990), and subsequently others, deal with only unsaturated zone, while Takeuchi et al. (2008) developed a numerical model which can solve the mixed form of Richards equation for saturated zone as well as for unsaturated zone.

For parameter identification problems, the earlier works have commonly used the well-defined models describing the soil hydraulic properties by a fixed-form function, like the van Genuchten-Mualem model (Mualem 1976, van Genuchten 1980). The fixed-form functions make the inverse method relatively easy-to-handle due to the limited number of parameters to be determined. Bitterlich et al. (2004) indicated, however, the two major drawbacks in employing this type of the function: (a) systematic errors in the fit of the retention function may propagate into the estimated conductivity function in cases where the selected fixed-form function is not flexible enough to represent the actual hydraulic properties because the small number of parameters may be achieved in part by coupling the retention function with the conductivity function through common parameters, and (b) in the fixed-form function the degree of freedom has a global character in that parameter changes will affect the hydraulic functions over the entire pressure head or water content domain even if only one degree of freedom is modified. Bitterlich et al. (2004) thus proposed a free-form parameterization approach using piecewise polynomial functions as an 
alternative to the classic parametric approach. The approach proposed by Bitterlich et al. (2004) estimates the soil hydraulic properties from the laboratory experiments that cannot be performed under fully natural conditions. In application of the properties obtained to the in-situ soil, therefore, care must be taken. To eliminate this defect of the approach, Izumi et al. (2008, 2009) proposed a field-oriented approach for the inverse estimation of soil hydraulic properties based on the free-form parameterization.

The inverse modeling proposed by Izumi et al. (2008, 2009 ) is based on the $\psi$-based form and $\theta$-based form of Richards equation. This paper is associated with an inverse modeling for the mixed form using the free-form parameterization technique. Firstly, the forward problem (FP) employing the mixed form of Richards equation as the governing equation is defined. Because the saturated hydraulic conductivity could be definitely known, the inverse problem related to the unsaturated hydraulic conductivity is reduced to a problem of identifying the relative hydraulic conductivity (RHC). Secondly, RHC is represented by a free-form parameterized function which is a sequence of piecewise cubic spline functions over the whole effective saturation domain. Thirdly, the inverse problem of minimizing errors between the observed and computed values of the pressure head is formulated based on a simulation-optimization algorithm with the aid of the Levenberg-Marqurdt method to find optimal values of the parameters included in the RHC function. Finally, the inverse method developed is validated through applying it for in-situ soil column in an upland crop field and comparing the observed water movement with the computed one obtained from the forward simulation for the desorption process in that considering the hysteric phenomenon is not needed.

\section{Forward problem (FP)}

\subsection{Governing equation}

The seepage flow in variably saturated soil can be described by Richards equation which combines the mass conservation equation and the Darcy-Buckingham's law, and its mixed form is represented as follows (Huyakorn and Pinder, 1983).

$$
\phi \frac{\partial S_{\mathrm{w}}}{\partial t}+S_{\mathrm{w}} S_{\mathrm{s}} \frac{\partial \psi}{\partial t}=-\frac{\partial}{\partial z}\left(-K \frac{\partial h}{\partial z}\right)
$$

with

$$
\begin{gathered}
S_{\mathrm{s}}=\rho_{\mathrm{w}} g\left(\beta_{\mathrm{s}}+\phi \beta_{\mathrm{w}}\right) \\
K=K_{\mathrm{r}} K_{\mathrm{S}}
\end{gathered}
$$

where $\phi$ is the porosity, $S_{\mathrm{w}}$ the saturation, $S_{\mathrm{s}}$ the specific storage, $\psi$ the pressure head, $K$ the unsaturated hydraulic conductivity, $h(=\psi+z)$ the hydraulic head, $t$ the time, $z$ the height defined as positive upward, $\rho_{\mathrm{w}}$ the water density assumed to be constant here, $g$ the gravitational acceleration, $\beta_{\mathrm{s}}$ and $\beta_{\mathrm{w}}$ the compressibility coefficients of soil and water, respectively, $K_{\mathrm{r}}$ the relative hydraulic conductivity (RHC), and $K_{\mathrm{S}}$ the saturated hydraulic conductivity.
Eq.(1) is subject to the following initial and boundary conditions.

$$
\begin{gathered}
\psi(z, 0)=\psi_{0}(z) \quad \text { in } \quad \Omega \\
\psi(z, t)=\bar{\psi}(z, t) \quad \text { on } \quad \Gamma_{\mathrm{d}} \\
-K_{\mathrm{s}} K_{\mathrm{r}} \frac{\partial h}{\partial z}=\bar{q}(z, t) \quad \text { on } \quad \Gamma_{\mathrm{n}}
\end{gathered}
$$

where $\psi_{0}(z)$ is the initial value of the pressure head in the space domain $\Omega, \bar{\psi}(z, t)$ the value of the pressure head on the Dirichlet boundary $\Gamma_{\mathrm{d}}$, and $\bar{q}(z, t)$ the water flux on the Neumann boundary $\Gamma_{\mathrm{n}}$.

Given that the coefficients of the derivatives in Eq.(1) is well parameterized or related to the unknown variables $\psi$, such a equations system can be numerically solved with respect to $\psi$ by the combined use of the standard Galerkin finite element method and the fully implicit time-marching scheme mentioned below. This forward solution procedure is embedded in the parameter optimization process.

\subsection{Parameterization of soil hydraulic properties}

To represent a constitutive relation between the volumetric water content and pressure head, or to obtain a soil water retention curve, the van Genuchten model (van Genuchten, 1980) is employed which is described as follows.

$$
S_{\mathrm{e}}=\frac{\theta-\theta_{\mathrm{r}}}{\theta_{\mathrm{s}}-\theta_{\mathrm{r}}}=\frac{1}{\left(1+(\alpha|\psi|)^{n_{\mathrm{vg}}}\right)^{m_{\mathrm{vg}}}}
$$

where $S_{\mathrm{e}}$ is the effective saturation, $\theta$ the volumetric water content, $\theta_{\mathrm{r}}$ the residual water content, $\theta_{\mathrm{s}}$ the saturated water content, and $\alpha, m_{\mathrm{vg}}$ and $n_{\mathrm{vg}}$ the unknown parameters, the last two being related as $m_{\mathrm{vg}}=1-1 / n_{\mathrm{vg}}$. To determine values of the parameters in Eq.(7) so as to best fit the observed $\theta-\psi$ relations, the least-square approach is used.

For functional representation of RHC, the free-form parameterization method is employed. For such a parameterization, the relation must be expressed by a function which is continuously differentiable over the whole effective saturation domain of interest. For this, as shown in Figure 1, the effective saturation domain is partitioned into $(I-1)$ subdomains with $I$ nodes ( $I$ denotes the degrees of freedom of the parameterization), and the function for $K_{\mathrm{r}}^{\mathrm{FFP}}\left(S_{\mathrm{e}}\right)$ over the whole domain $\left[S_{\mathrm{e}}\left(\theta_{\mathrm{r}}\right)=0, S_{\mathrm{e}}\left(\theta_{\mathrm{s}}\right)=1\right]$ is expressed by a sequence of piecewise cubic spline functions each of which is locally defined over a confined sub-domain bounded with two nodes. Thus the function defined over the $i$ th sub-domain of $\left[S_{\mathrm{e}, i}, S_{\mathrm{e}, i+1}\right], K_{\mathrm{r}, i}\left(S_{\mathrm{e}}\right)$ is described as follows.

$$
K_{\mathrm{r}}^{\mathrm{FFP}}\left(S_{\mathrm{e}}\right)=\sum_{i=1}^{I-1} K_{\mathrm{r}, i}\left(S_{\mathrm{e}}\right)
$$

with

$$
K_{\mathrm{r}, i}\left(S_{\mathrm{e}}\right)=\left\{\begin{array}{lr}
a_{i}^{k}+b_{i}^{k}\left(S_{\mathrm{e}}-S_{\mathrm{e}, i}\right)+c_{i}^{k}\left(S_{\mathrm{e}}-S_{\mathrm{e}, i}\right)^{2} \\
+d_{i}^{k}\left(S_{\mathrm{e}}-S_{\mathrm{e}, i}\right)^{3}, & S_{\mathrm{e}} \in\left[S_{\mathrm{e}, i}, S_{\mathrm{e}, i+1}\right] \\
0, & S_{\mathrm{e}} \notin\left[S_{\mathrm{e}, i}, S_{\mathrm{e}, i+1}\right]
\end{array}\right.
$$




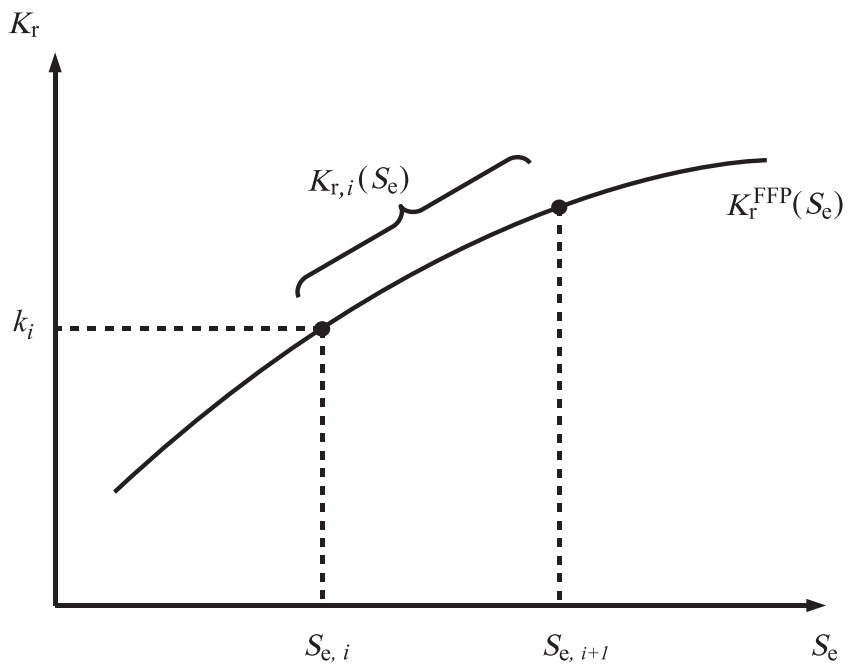

Figure 1: Free-form parameterization

where $a_{i}^{k}, b_{i}^{k}, c_{i}^{k}$ and $d_{i}^{k}$ are coefficients in the cubic splines, and $i(1 \leq i \leq I)$ a nodal number. Hereinafter, the values of $K_{\mathrm{r}}^{\mathrm{FFP}}\left(S_{\mathrm{e}}\right)$ at a node $i$ are simply denoted by $k_{i}$. Since, as well known, the relative hydraulic conductivity monotonously increases with the increasing saturation, Eq.(8) must be identified so that the following constraints are satisfied.

$$
k_{i} \leq k_{i+1}
$$

\subsection{Time-marching scheme}

The governing equation is discretized by a standard Galerkin finite element method for space and a finite difference method for time. As the time-marching scheme, the modified Picard method proposed by Celia et al. (1990) is used. If the superscripts $m$ and $n$ stand for an iteration step and a time level, respectively, then the Picard iteration scheme can be written as

$$
\begin{gathered}
\frac{S_{\mathrm{w}}^{n+1, m+1}-S_{\mathrm{w}}^{n}}{\Delta t}+\frac{S_{\mathrm{w}}^{n+1, m} S_{\mathrm{s}}}{\phi} \frac{\psi^{n+1, m}-\psi^{n}}{\Delta t} \\
-\frac{1}{\phi}\left(\frac{\partial}{\partial z}\left(K^{n+1, m} \frac{\partial \psi^{n+1, m}}{\partial z}\right)-\frac{\partial K^{n+1, m}}{\partial z}\right)=0
\end{gathered}
$$

According to Celia et al. (1990), $S_{\mathrm{w}}^{n+1, m+1}$ is expanded with respect to $\psi$ by Taylor series expansion, ignoring the higher order, as follows.

$$
\begin{gathered}
S_{\mathrm{w}}^{n+1, m+1}=S_{\mathrm{w}}^{n+1, m}+\left.\frac{d S_{\mathrm{w}}}{d \psi}\right|^{n+1, m}\left(\psi^{n+1, m+1}-\psi^{n+1, m}\right) \\
=S_{\mathrm{w}}^{n+1, m}+\frac{C_{\mathrm{w}}^{n+1, m}}{\phi}\left(\psi^{n+1, m+1}-\psi^{n+1, m}\right)
\end{gathered}
$$

where $C_{\mathrm{w}}$ is the specific water content. Substituting the truncated series Eq.(12) into Eq.(11) yields

$$
\begin{gathered}
\psi^{n+1, m+1}=\psi^{n+1, m}+\Delta t \frac{\phi}{C_{\mathrm{w}}^{n+1, m}}\left(-\frac{S_{\mathrm{w}}^{n+1, m}-S_{\mathrm{w}}^{n}}{\Delta t}\right. \\
\left.-\frac{S_{\mathrm{w}}^{n+1, m} S_{\mathrm{s}}}{\phi} \frac{\psi^{n+1, m}-\psi^{n}}{\Delta t}+\frac{1}{\phi}\left(\frac{\partial}{\partial z}\left(K^{n+1, m} \frac{\partial \psi^{n+1, m}}{\partial z}\right)+\frac{\partial K^{n+1, m}}{\partial z}\right)\right)
\end{gathered}
$$

When a soil domain of interest is saturated, $S_{\mathrm{w}}=$ const. $=$ 1. Therefore, the first term on the left-hand side of Eq.(1) disappears, and the equation becomes as follows.

$$
S_{\mathrm{s}} \frac{\partial \psi}{\partial t}=-\frac{\partial}{\partial z}\left(-K \frac{\partial h}{\partial z}\right)
$$

Employing the modified Picard scheme, Eq.(14) can be written in the time-marching form as follows.

$$
\psi^{n+1, m+1}=\psi^{n}+\Delta t \frac{1}{S_{\mathrm{S}}}\left(\frac{\partial}{\partial z}\left(K^{n+1, m} \frac{\partial \psi^{n+1, m}}{\partial z}\right)+\frac{\partial K^{n+1, m}}{\partial z}\right)
$$

\section{Inverse problem (IP)}

\subsection{Formulation of optimization problem}

Solving the inverse problem (IP) is defined as optimally deciding a set of unknown variables, $\boldsymbol{k}=\left\{k_{i}, 1 \leq i \leq I\right\}$. The objective function is then defined as the total least squares error integrated over space and time between the solution of FP $\left(\psi^{\mathrm{com}}(\boldsymbol{k})\right)$ and the observed data $\left(\psi^{\mathrm{obs}}\right)$, and therefore, written as

$$
J(\hat{\boldsymbol{k}})=\min J(\boldsymbol{k}), \quad \boldsymbol{k} \in K_{\mathrm{ad}}
$$

with

$$
\begin{gathered}
J(\boldsymbol{k})=\frac{1}{2} \sum_{l=1}^{L}\left\{f_{l}(\boldsymbol{k})\right\}^{2} \\
f_{l}(\boldsymbol{k})=w_{l}\left(\psi_{l}^{\mathrm{com}}(\boldsymbol{k})-\psi_{l}^{\mathrm{obs}}\right)
\end{gathered}
$$

where $\hat{\boldsymbol{k}}$ is a set of $\boldsymbol{k}$ optimal solutions, $K_{\mathrm{ad}}$ an admissible set of $\boldsymbol{k}, L$ the total number of observed data available in space and time, and $w_{l}$ (normally, taken as unity) a weighting factor. The coefficient of $1 / 2$ in Eq.(17) is an additive constant to cancel the differential coefficient obtained from differentiating the sum of squares.

In the process of minimizing Eq.(17) subject to Eq.(10), the decision variables, $\boldsymbol{k}$, are iteratively updated while step by step solving FP with their assumed or previously estimated values. In this respect, identification of the function (Eq.(8)) requires a sort of simulation-optimization technique.

\subsection{Optimization Algorithm}

For the optimization algorithm to search for the set of the optimal solutions, $\hat{\boldsymbol{k}}$, Levenberg-Marquardt method, which is a modified Gauss-Newton method, is adopted. With this method, the unknown parameters are step by step updated with the following search sequence through the iteration.

$$
\boldsymbol{k}^{(\gamma+1)}=\boldsymbol{k}^{(\gamma)}+\Delta \boldsymbol{k}^{(\gamma)}
$$

with

$$
\begin{gathered}
\Delta \boldsymbol{k}^{(\gamma)}=-\left(\boldsymbol{H}^{(\gamma)}+\eta \boldsymbol{I}\right)^{-1} \nabla \boldsymbol{J}^{(\gamma)} \\
\boldsymbol{H}^{(\gamma)}=\left[\sum_{l=1}^{L} \frac{\partial f_{l}^{(\gamma)}}{\partial k_{i}} \frac{\partial f_{l}^{(\gamma)}}{\partial k_{j}}\right]
\end{gathered}
$$

where $\gamma$ is an iteration number, $\eta$ a coefficient, and $\boldsymbol{I}$ the $I \times I$ unit matrix. When $\eta$ is equal to zero, $\Delta \boldsymbol{k}^{(\gamma)}$ reduces to the 
Gauss-Newton direction. On the other hand, when tends to infinity, $\Delta \boldsymbol{k}^{(\gamma)}$ turns to the steepest descent direction and size of $\Delta \boldsymbol{k}^{(\gamma)}$ tends to zero. Therefore, $\eta$ is taken as zero for an initial value, and if $J\left(\boldsymbol{k}^{(\gamma+1)}\right)<J\left(\boldsymbol{k}^{(\gamma)}\right)$ is not satisfied, then value of $\eta$ is increased and $\Delta \boldsymbol{k}^{(\gamma)}$ is recomputed with Eq.(20) until $J\left(\boldsymbol{k}^{(\gamma+1)}\right)<J\left(\boldsymbol{k}^{(\gamma)}\right)$ is satisfied (Sun, 1994).

\section{Validation}

An in-situ application to the test soil (sandy soil) in an upland crop field in Miyoshi, Aichi Prefecture, Japan, is implemented to examine validity of the inverse method described above. The validity is judged referring to reproducibility of the forward solutions obtained from the calibrated model.

\subsection{Observation system}

The data necessary for identification of RHC is acquired by the observation system made up of different three sets of instruments (tensiometer and soil moisture probe), which are buried at different three depths in a soil column (Figure 2). At individual depths, serial data of pressure head and volumetric water content are observed which are necessary to identify the soil water retention property. The observed pressure heads at both top and bottom of the soil column are also used as Dirichlet boundary values in solving FP based on the governing equation. The pressure head observed at the intermediate depth $(-20 \mathrm{~cm}$ deep) is used to judge the fitness of estimated unknown parameters (or RHC function) in an optimization process.

The targeted domain is the surface soil of $20 \mathrm{~cm}$ thick between $-10 \mathrm{~cm}$ and $-30 \mathrm{~cm}$ deep, where soil alternates between saturated and unsaturated situations with frequency, and is discretized into four equal elements with five nodes for numerical computations. The time-varying pressure heads and volumetric water contents at the three depths of $-10 \mathrm{~cm},-20$ $\mathrm{cm}$ and $-30 \mathrm{~cm}$ are automatically logged at intervals of 10 minutes.

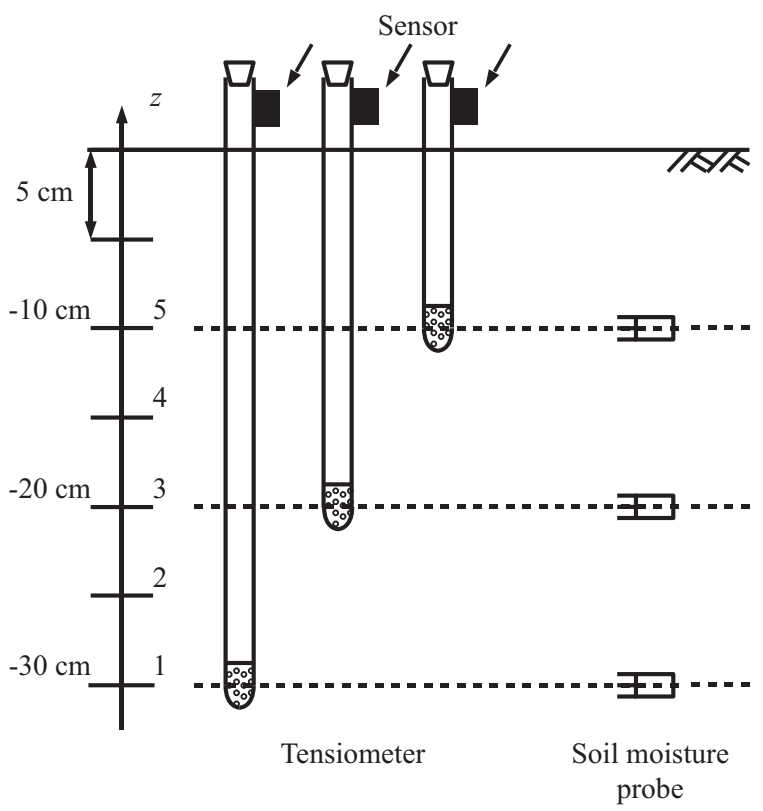

Figure 2: Observation system, and discretization of targeted domain

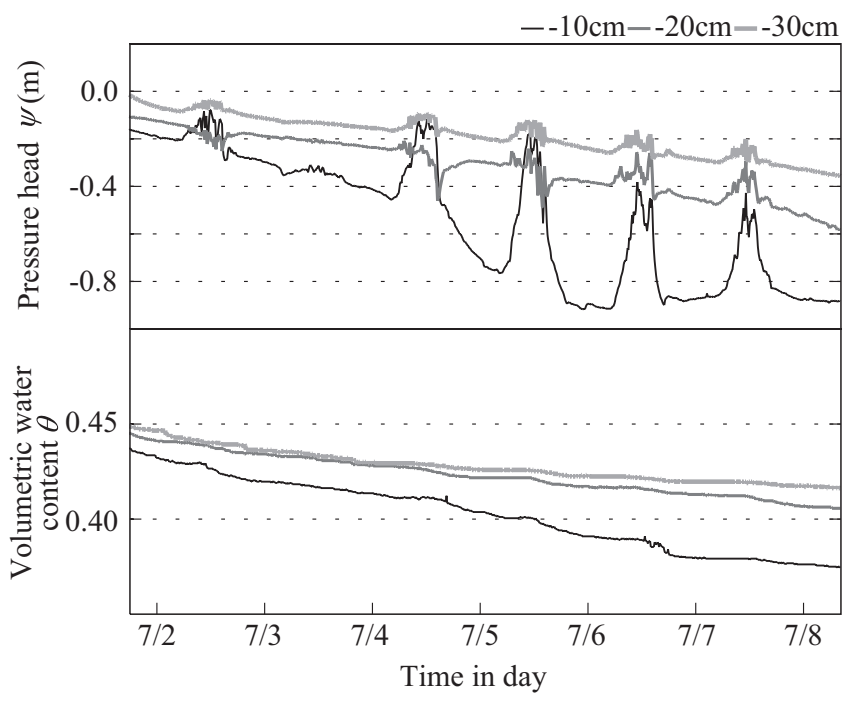

Figure 3: Observed data for validation

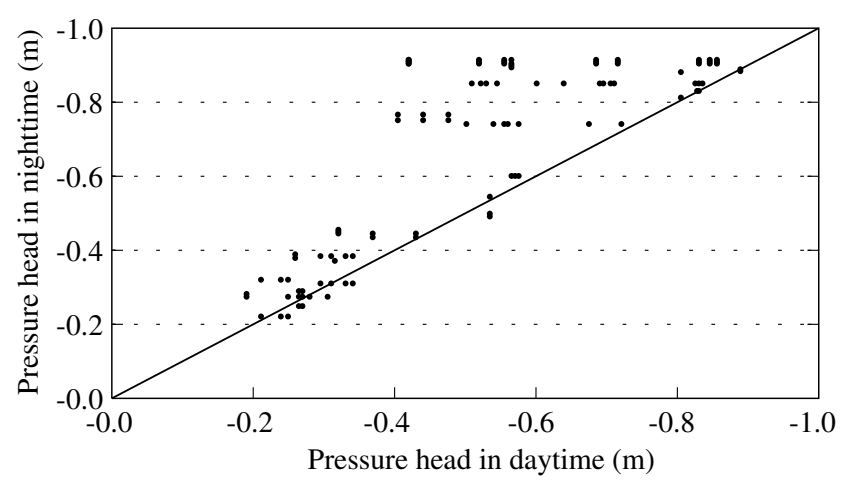

Figure 4: Correlation between pressure heads in daytime and nighttime for the same volumetric water content

A one-way process of desorption is considered for estimation of the soil hydraulic properties to exclude the hysteric phenomenon, as in most of the preceding studies associated with parameter identification. The data observed during the no-rainfall period (or desorption period) of July 1 to 9, 2008, used as basic data for calibration of the numerical model as well as for validation of the inverse method presently developed, are illustrated in Figure 3. From Figure 3, it can readily be seen that the pressure head involves periodic daily variations while declining in time, having a distinct difference from the volumetric water content. Even if the volumetric water contents in daytime and nighttime are the same, therefore, the pressure heads are different as shown in Figure 4. This result is against the theory that there should be a oneto-one correspondence between the pressure head and the volumetric water content. As Inoue et al. (2004) reported, this is because the sensing part of this type of a tensiometer (UIZ-SMT, UIZIN Co., Ltd.) is put near the top of the tube, and therefore the readings of the pressure head are significantly affected by air temperature. Thus, as described later, identification of RHC function is comparatively done considering another data series of the pressure head in which temperature-dependency of the pressure heads in the daytime of 6:00 to 18:00 is removed by means of the linear interpolation method. 


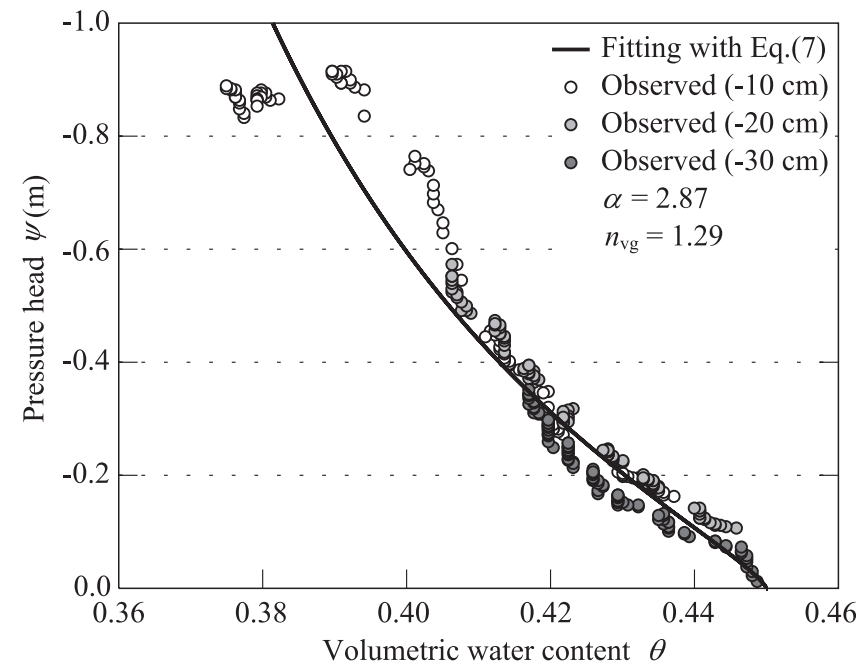

Figure 5: Soil water retention property

\subsection{Identification of water retention property}

Identifying RHC function must be preceded by fixing the form of the soil water retention curve expressed by Eq.(7). The saturated water content $\theta_{\mathrm{S}}$ is defined as the value of $\theta$ in case that $\psi$ is equal to zero, and the residual water content $\theta_{\mathrm{r}}$ is defined as the minimum value in all the observed serial data of $\theta$. Thus, straightforwardly from the data observed, $\theta_{\mathrm{s}}$ and $\theta_{\mathrm{r}}$ can be identified to be 0.45 and 0.22 , respectively. By use of the least squares method proposed by Takeshita and Kohno (1993), the remaining parameters $\alpha$ and $n_{\mathrm{vg}}$ are determined so that Eq. (7) can be best fit to the observed relations between $\psi$ and $\theta$. Note that in this curve-fitting the temperature-dependent $\theta-\psi$ relations observed during the daytime of 6:00 to 18:00 are not considered. Eventually the values of $\alpha$ and $n_{\mathrm{vg}}$ are identified to be 2.87 and 1.29, respectively, to have a best fitting curve as shown in Figure 5.

\subsection{Identification of RHC and validation of the method}

As mentioned above, the time-varying pressure head obtained from a field observation is significantly affected by air temperature. Different two data sets of time-varying pressure head are thus considered for identifying RHC (or calibrating the model) by free-form parameterization and examining solution reproducibility (accuracy) of the model so calibrated. One is the observed data as they are, and the other is the corrected data which can be obtained from filtering out daytime temperature-dependent noises from the observed data, or from assuming that the pressure head varies linearly from the observed head at 6:00 to that at 18:00. These are referred to as $<$ Data $\mathrm{A}>$ and $<$ Data $\mathrm{B}>$, respectively.

The value of $K_{\mathrm{s}}$ is given as $4.5 \times 10^{-4} \mathrm{~m} / \mathrm{s}$ from the result of the laboratory tests for the soils sampled from the site. In application of Eq.(18), all $w_{l}$ are equalized to unity.

The results for $<$ Data $\mathrm{A}>$ and $<$ Data $\mathrm{B}>$ are shown in Figures 6 and 7, respectively, illustrating the identified RHC function $K_{\mathrm{r}}^{\mathrm{FFP}}$ and the solution reproducibility. The solution reproducibility is examined through comparing the computed $\psi^{\text {com }}$ (or the forward solution with the identified $K_{\mathrm{r}}^{\mathrm{FFP}}$ ) with the non-corrected (observed) or corrected $\psi^{\mathrm{obs}}$, and measured in terms of the absolute error $E^{\mathrm{com}}=\left|\psi^{\mathrm{com}}-\psi^{\mathrm{obs}}\right|$. Values of the objective function $J(k)$, which are reached in the simulation-optimization processes for identifying RHC function and thus could be a global indicator of solution reproducibility, are listed in Table 1.

From Figure 6 (b), it can be seen that the model calibrated for $<$ Data $A>$ produces the forward solutions in partially good agreement with the original observed data. From Figure 7 (b), on the other hand, it is recognized that the model calibrated for $<$ Data $B>$ reproduces the corrected observed data in closer agreement. Table 1 also demonstrates the same in a quantitative sense. It is thus concluded that the method presently developed could be a viable tool for finding RHC in a functional form, fulfilling its function with better performance if air-temperature dependency of the pressure head variation is appropriately eliminated.

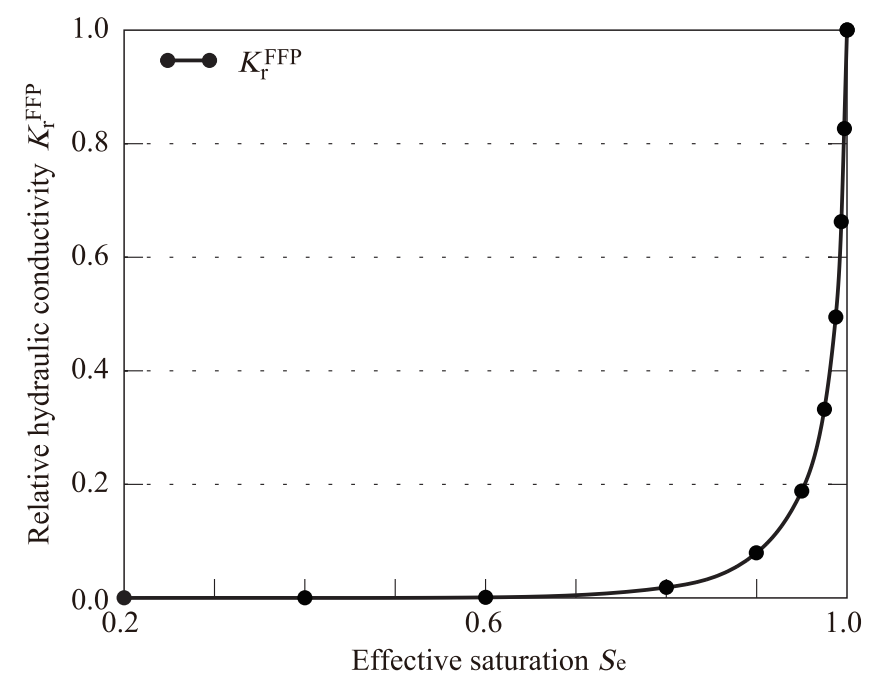

(a)

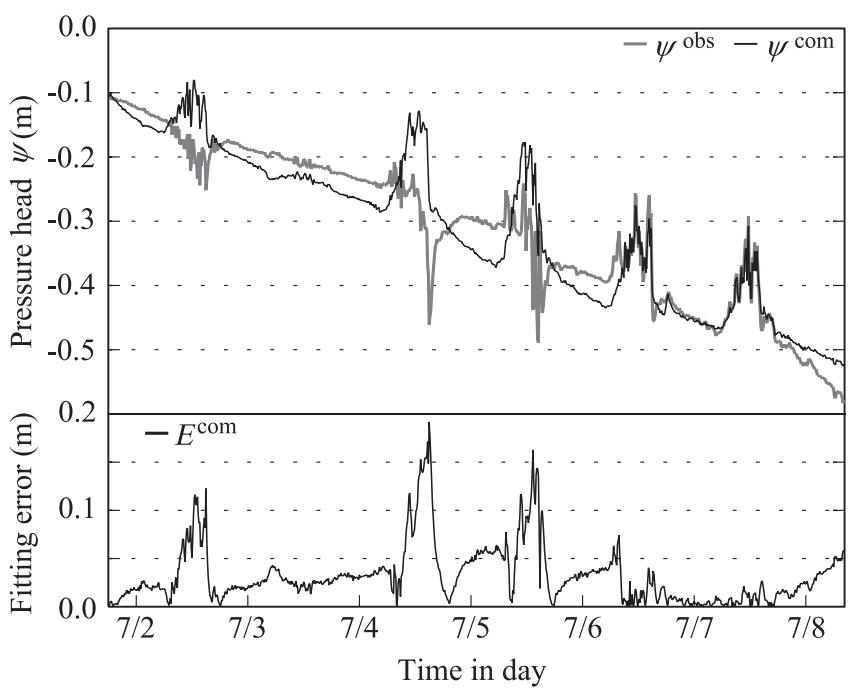

(b)

Figure 6: (a) Identified RHC and (b) Reproducibility of FP solution for $<$ Data $A>$ 


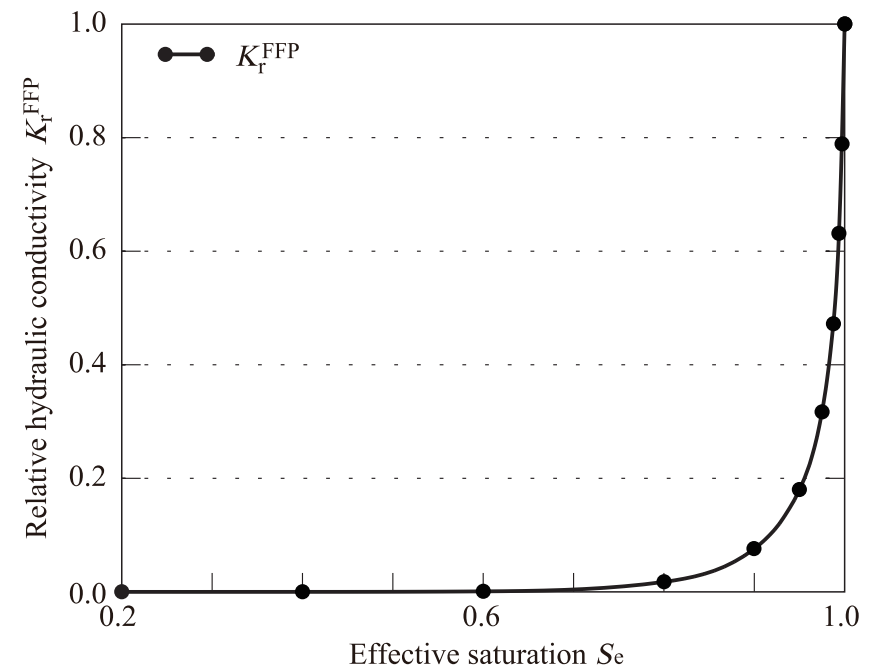

(a)

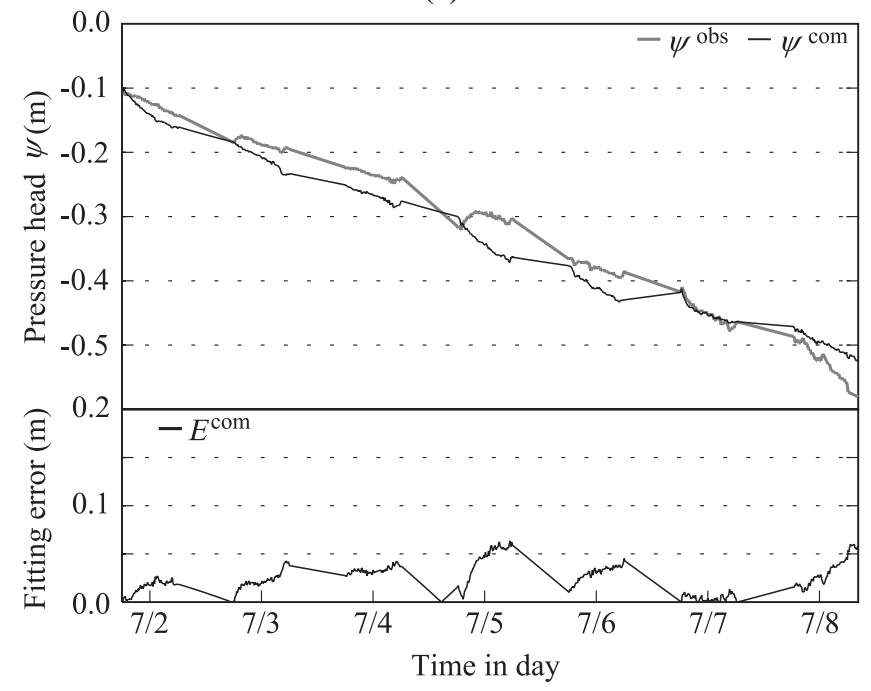

(b)

Figure 7: (a) Identified RHC and (b) Reproducibility of FP solution for $<$ Data $B>$

Table 1: Minimum values of $J(\boldsymbol{k})$ in simulation-optimization

\begin{tabular}{cc}
\hline Data type & $J(\boldsymbol{k})$ \\
\hline$<$ Data $\mathrm{A}>$ & 1.88 \\
$<$ Data $\mathrm{B}>$ & 0.737 \\
\hline
\end{tabular}

\section{Conclusions}

A method for solving the inverse problem associated with variably saturated seepage flow has been developed. The mixed-form Richards equation which provides massconservative solutions to saturated-unsaturated seepage flow problem is considered as the governing equation which describes the forward problem. Examinations for validation of the method developed show that the relative hydraulic conductivity which is a major hydraulic property of the governing equation system, expressed by a free-form sequential piecewise cubic spline function, could successfully be identified through determining the values of the coefficients of the function with the aid of the simulation-optimization technique. The results also show that the forward solution model with identified relative hydraulic conductivity could reproduce actual one-way desorption process with higher accuracy when air-temperature dependency of the observed pressure head variation is appropriately eliminated.

\section{References}

[1] Bitterlich, S., Durner, W., Iden, S.C. and Knabner, P. (2004): Inverse estimation of the unsaturated soil hydraulic properties from column outflow experiments using free-form parameterizations, Vadose Zone Journal, 3, pp.971-981.

[2] Celia, M.A., Bouloutas, E.T. and Zarba, R.L. (1990): A general mass-conservative numerical solution for the unsaturated flow equation, Water Resources Research 26(7), pp.14831496.

[3] Huang, K., Mohanty, B.P. and van Genuchten, M.T. (1996): A new convergence criterion for the modified Picard iteration method to solve the variably saturated flow equation, Journal of Hydrology 178, pp.69-91.

[4] Huyakorn, P.S. and Pinder, G.F. (1983): Computational Methods in Subsurface Flow, Academic Press, 473p.

[5] Inoue, M., Morii, T., Nishimura, T. and Fujimaki, H. (2004): In-situ determination of unsaturated hydraulic conductivity using profile probe, Transactions of the Japanese Society Irrigation, Drainage and Reclamation Enginnering 231:39-45 (in Japanese with English abstract)

[6] Izumi, T., Takeuchi, J., Kawachi, T., Unami, K. and Maeda, S. (2008): An inverse method to estimate soil hydraulic properties in saturated-unsaturated groundwater flow model, Journal of Rainwater Catchment Systems, 13(2), pp.23-28.

[7] Izumi, T., Takeuchi, J., Kawachi, T. and Fujihara, M. (2009): An inverse method to estimate unsaturated hydraulic conductivity in seepage flow in non-isothermal soil, Transactions of the Japanese Society Irrigation, Drainage and Rural Enginnering, 264, pp.35-42.

[8] Mualem, Y. (1976): A new model for predicting the hydraulic conductivity of unsaturated porous media, Water Resources Research, 12(3), pp.513-522.

[9] Sun, N-Z. (1994): Inverse Problems in Groundwater Modeling , Kluwer Academic Publishers, 337p.

[10] Takeshita, Y. and Kohno, I. (1993): A method to predict hydraulic properties for unsaturated soils and its application to observed data, Ground Engineering; Journal of Chugoku Branch, JGS, 11(1), pp.95-113 (in Japanese with English abstract).

[11] Takeuchi, J., Kawachi, T. and Izumi, T. (2008): Explicit scheme for mixed form of Richards equation. Proceedings of the Japanese Society of Irrigation, Drainage and Rural Enginnering 3-12 (in Japanese)

[12] van Genuchten, M.Th. (1980): A closed-form equation for predicting the hydraulic conductivity of unsaturated soils, Soil Science Society of American Journal, 44, pp.892-898. 